\title{
Rôle de la végétation dans la protection contre l'érosion hydrique de surface
}

\author{
Freddy Rey ${ }^{\mathrm{a}, *}$, Jean-Louis Ballais ${ }^{\mathrm{b}}$, Alain Marre ${ }^{\mathrm{c}}$, Georges Rovéra ${ }^{\mathrm{d}}$ \\ a Cemagref, UR EPM, 2, rue de la Papeterie, BP 76, 38402 St-Martin-d'Hères cedex, France \\ ${ }^{\mathrm{b}}$ UMR ESPACE CNRS, université de Provence, 29, av. Robert-Schuman, 13621 Aix-en-Provence cedex, France \\ c Département de Géographie, université de Reims-Champagne-Ardenne, GREURCA, 57, rue Pierre-Taittinger, \\ 51096 Reims cedex, France \\ d Institut de Géographie Alpine, SEIGAD, université Joseph-Fourier, 14bis, av. Marie-Reynoard, 38100 Grenoble, France \\ Reçu le 20 juin 2003 ; accepté après révision 15 mars 2004 \\ Disponible sur Internet le 10 juin 2004 \\ Présenté par Georges Pédro
}

\begin{abstract}
Résumé
Le présent article se propose de faire le point sur les connaissances actuelles quant au rôle de la végétation dans la protection contre l'érosion hydrique de surface. Il expose, dans un premier temps, les mécanismes d'action de la végétation vis-à-vis des processus d'érosion et de sédimentation. Dans un deuxième temps, il présente des résultats de recherches sur l'efficacité des différentes formations végétales et l'importance du taux de couverture végétale pour la lutte contre l'érosion de ravines. Pour citer cet article : F. Rey et al., C. R. Geoscience 336 (2004).
\end{abstract}

(C) 2004 Académie des sciences. Publié par Elsevier SAS. Tous droits réservés.

\section{Abstract}

Role of vegetation in protection against surface hydric erosion. This paper is a review on the current knowledge concerning the role of vegetation in protection against surface hydric erosion. It first explains the mechanisms of vegetation action in relation to processes of erosion and sedimentation. Secondly, it presents the results of researches carried out on the efficiency of different vegetation structures and the significance of the rate of vegetation cover for combating gully erosion. To cite this article: F. Rey et al., C. R. Geoscience 336 (2004).

(C) 2004 Académie des sciences. Publié par Elsevier SAS. Tous droits réservés.

Mots-clés : érosion ; génie biologique ; hydrologie ; sédiment ; végétation

Keywords: erosion; bioengineering; hydrology; sediment; vegetation

\section{Abridged English version}

This paper is a review of current knowledge con-

* Auteur correspondant.

Adresse e-mail : freddy.rey@ cemagref.fr (F. Rey). cerning the role of vegetation in protection against surface hydric erosion. 
It first explains the mechanisms of vegetation action in relation to erosion and sedimentation processes. Firstly, vegetation can protect soils against erosive factors. It can act as a hydrological catchment regulator, by intercepting raindrops and increasing water infiltration in soils. The results of this regulation are a decrease of flow quantity, concentration and yield, and thus of erosive activity. It can provide mechanical protection, by reducing the energy of raindrops and 'splash' effects. It can also play a role of thermal regulation, by reducing daily temperature variations. Moreover, vegetation allows soil fixation by roots. Secondly, vegetation can play a role in sedimentation by trapping and retaining some of the sediment eroded within a catchment. There is an effect of flow filtration through vegetation. These flows deposit sediment as a function of the reduction of their transport energy. Deposits created by vegetation barriers favour colonisation by vegetation and the plants of vegetation barriers can also develop on the deposited sediment, and then colonize trapped sediment. Consequently, large quantities of eroded sediment inside catchments are trapped and do not reach catchment exits.

The paper then presents the results of research carried out on the efficiency of different vegetation structures. The effect of vegetation may differ depending on plant structures, type of vegetation and soil use. Some authors state that forest is the best protection against erosion in mountain lands. Others have observed that shrubs or low vegetation can play a protective role similar to that of trees. The role of herbaceous vegetation is often denigrated, whereas it can be very efficient in fighting against erosion. For the same plant structure, the efficiency of protection against erosion may depend on the plant species, in particular on their morphology and dynamics.

Finally, this article provides elements on the significance of the rate of vegetation cover in erosion and sedimentation control highlighted by comparison between catchments with different plant covers. It is generally admitted that erosion occurs in bare zones, whereas vegetated ones are protected. Studies carried out at the catchment scale have shown that erosion decreases when plant cover increases, yet several studies have shown that the sediment yield of catchments and gullies does not vary as a linear function with plant cover. The increase of erosion is generally observed in catchments where fires or forestry operations have caused vegetation to disappear. Natural disasters such as windstorms can also lead to the renewal of erosion. On the contrary, revegetation operations and the natural installation of vegetation on eroded soils generally cause erosion to decrease.

\section{Introduction}

L'érosion de surface réunit tous les processus qui affectent, sur une faible profondeur, les sols, les formations superficielles et/ou le substratum géologique. Elle est ainsi essentiellement due à l'action des eaux courantes, appelée aussi «érosion hydrique », qui comprend des formes de ruissellement et de petites coulées boueuses de quelques centimètres à quelques décimètres d'épaisseur, mais elle exclut tous les grands mouvements de terrain qui peuvent avoir des profondeurs de plusieurs mètres. Elle comprend deux phases qui se succèdent : la première phase correspond à l'ablation du substrat; la deuxième correspond au transport et au dépôt de sédiments érodés.

L'érosion hydrique de surface constitue un phénomène naturel, aux conséquences préoccupantes dans beaucoup de régions du monde (par exemple, [40]). La maîtrise de l'érosion hydrique de surface représente alors un défi majeur sur les espaces où se localisent des enjeux socio-économiques [39]. Il est généralement admis que la végétation peut constituer un moyen de lutte efficace contre elle et il existe ainsi une grande quantité de références à ce sujet dans la littérature scientifique. Il a donc paru intéressant de faire le point sur les connaissances quant au rôle de la végétation dans la protection contre l'érosion hydrique de surface, dans diverses conditions de climat et à différentes échelles spatiales. Cet article tente de dresser un panorama général des diverses études menées dans ce domaine, en présentant d'abord les connaissances sur les mécanismes d'action de la végétation vis-à-vis des différents types de processus érosifs. Ensuite, les résultats d'investigations portant sur l'efficacité de différentes formations végétales sur l'érosion hydrique de surface sont présentés. Enfin, les résultats des recherches concernant l'importance du taux de couverture végétale sont exposés.

\section{Les mécanismes d'action de la végétation}

La végétation peut intervenir contre l'érosion hydrique de surface de deux manières principales : d'une 
part, elle peut empêcher l'ablation du substrat, d'autre part, elle peut favoriser la sédimentation, en retenant les sédiments érodés plus à l'amont.

\subsection{Rôle de la végétation contre l'ablation}

La végétation protège les sols de l'ablation par réduction de l'énergie des agents érosifs et maintien des sols.

\subsubsection{Réduction de l'énergie de l'érosion pluviale}

La végétation permet de réduire l'énergie de l'érosion pluviale en jouant un rôle d'interception des gouttes de pluie, grâce aux parties aériennes des plants. Cette interception est fonction de la densité de la surface foliaire [15] et de la structure du couvert végétal [60]. En forêt, les précipitations inférieures à $2 \mathrm{~mm}$ en $24 \mathrm{~h}$ sont presque entièrement interceptées; au-delà, l'interception diminue lorsque l'intensité de la pluie augmente, selon une fonction logarithmique. Pour une même essence, l'interception augmente avec la densité et la surface terrière [24]. Par son couvert, la végétation joue ainsi un rôle de protection mécanique : elle permet de diminuer l'énergie cinétique des gouttes de pluie et de réduire l'effet de splash [23], surtout grâce aux litières et buissons, dont le rôle est plus important que celui de la canopée aérienne.

\subsubsection{Réduction de l'énergie du ruissellement}

Au sol, la végétation permet de lutter contre le ruissellement, en augmentant l'infiltration de l'eau [13]. Elle constitue pour cela un écran à la surface du sol, barrant le cheminement des filets d'eau. L'architecture individuelle des plants ou des associations de plants joue à ce titre un rôle important. De plus, la végétation améliore les propriétés physiques et chimiques des sols. C'est notamment le cas pour la végétation feuillue, considérée comme une formation végétale «améliorante» pour les sols, ou en général pour les formations végétales denses [51]. Zordia [60] précise que la pédogenèse sous forêt est plus importante que sous n'importe quelle autre formation végétale, car la biomasse, l'activité biologique et l'enracinement sont plus importants. Giordano [24] rapporte qu'un sol forestier favorise l'infiltration de l'eau dans le sol plus qu'une autre occupation du sol. Une litière forestière hétérogène et perméable peut ainsi absorber et retenir plus de $10 \mathrm{~mm}$ d'eau. Les sols sont plus poreux et favorisent, de ce fait, l'infiltration de l'eau. Ils sont alors à même de stocker de plus grandes quantités d'eau, jouant un rôle d'éponge, plus ou moins important selon l'état de saturation des sols. Dunne et al. [20] indiquent que le taux d'infiltration des eaux de ruissellement pour un sol dépend de la surface drainée à l'amont. Une partie de l'eau infiltrée peut ensuite être restituée sous forme de ruissellement hypodermique, mais avec un certain retard [41].

La régulation hydrologique jouée par la végétation a ainsi pour effet de diminuer la quantité, la concentration et le débit du ruissellement [14,32]. Giordano [24] rapporte qu'entre des terrains nus et une forêt, le ruissellement diminue et le temps d'écoulement de la pluie augmente jusqu'à 500 fois. Richard et Mathys [46], en faisant la comparaison entre un bassin versant dénudé et un bassin versant végétalisé, ont observé une augmentation du seuil minimal de pluie pour obtenir un ruissellement et, de ce fait, une réduction de la fréquence des crues. Les pointes de crues sont retardées d'au moins une demi-heure et réduites dans un rapport de 5 à 10. Le tarissement est également plus lent. Combes et al. [14] expliquent que, par rapport à des terrains dénudés, la forêt peut réduire d'au moins $80 \%$ la pointe de crue et d'au moins $50 \%$ le volume ruisselé rapidement. Elle peut tripler la rétention initiale avant le début d'un écoulement. Elle peut multiplier par cinq la capacité maximale d'infiltration pendant une crue. Il existe, cependant, des seuils d'efficacité en ce qui concerne l'hydrologie : l'influence de la forêt est moindre pour les événements pluvieux longs ou abondants [60]. En effet, une fois le sol saturé, la forêt ne joue qu'un rôle réduit dans le ruissellement. McIvor et al. [34] ont ainsi observé que, pour des pluies de plus de $100 \mathrm{~mm}$ en $24 \mathrm{~h}$ avec une intensité supérieure à $45 \mathrm{~mm} \mathrm{~h}^{-1}$, la végétation ne jouait pas un grand rôle dans la réduction du ruissellement. De plus, le rôle de la végétation est à pondérer selon la saison : en hiver en zone tempérée, et sous des espèces à feuilles caduques, son action est nettement ralentie [41].

\subsubsection{Maintien des sols}

La végétation permet de maintenir les sols grâce aux systèmes radiculaires. Les végétaux permettent, en effet, d'améliorer la cohésion des sols et donc de renforcer leurs propriétés mécaniques [37]. Sassa [52] explique que des racines pénétrant à $50 \mathrm{~cm}$ dans un sol peuvent doubler la consolidation de ce dernier. Cet effet de renforcement des sols grâce aux systèmes 
radiculaires a également été mis en évidence sur les berges végétalisées des cours d'eau [1].

La végétation peut aussi jouer un rôle de régulation thermique : elle permet d'atténuer les écarts quotidiens de températures, réduisant ainsi les effets de la gélifraction [50]. En particulier, la forêt peut jouer un rôle important de protection thermique : les températures maximales sous forêt sont en général inférieures de 2 à $4{ }^{\circ} \mathrm{C}$ par rapport à la campagne environnante, et les minimales supérieures de 1 à $2{ }^{\circ} \mathrm{C}$. Stott [55] a également observé le rôle de régulation thermique joué par une végétation recouvrant des berges de cours d'eau : les températures du sol sont supérieures de près de $4{ }^{\circ} \mathrm{C}$ pour des berges végétalisées par rapport à des berges dénudées.

\subsection{Rôle de la végétation en faveur de la sédimentation}

La végétation peut exercer un effet favorable sur la sédimentation des particules, grâce à des processus de piégeage et de rétention d'une partie des sédiments érodés à l'intérieur d'un bassin versant. Lee et al. [33] ont montré qu'il existe un effet de filtration des écoulements au travers de la végétation. Ces écoulements déposent les sédiments par suite de la réduction de leur énergie de transport. Bien que Beuselinck et al. [8] aient montré que la végétation est davantage susceptible de piéger les sédiments fins, d'autres études [35] ont montré que les sédiments grossiers pouvaient également être piégés par des obstacles végétaux. Des dépôts ont ainsi été observés à l'amont de barrières végétales, sur des pentes plus fortes que celles sans végétation [9].

L'utilisation de haies végétales sur terrasses pour favoriser la sédimentation a souvent été testée avec succès. Les haies végétales de vétiver (Vetiveria $s p$.) ont notamment souvent montré leur efficacité pour le piégeage des sédiments [17]. Ce piégeage a également été observé sur les berges des cours d'eau. Les accumulations de sédiments permettent alors de renforcer la stabilité des berges [56].

La continuité de la barrière végétale apparaît comme un facteur important pour l'efficacité du piégeage. Cela a été établi par :

- Meyer et al. [35] et Bochet et al. [9], qui ont montré que la morphologie du plant est importante, c'est-à-dire que l'espèce végétale joue un rôle prépondérant;

- Lee et al. [33] et Roose et Bertrand [48], qui ont mis en évidence le fait que l'efficacité du piégeage augmente avec la largeur - perpendiculairement à l'écoulement - de la barrière ;

- Abu-Zreig et al. [2], qui ont montré l'influence de la longueur - dans le sens de l'écoulement - de la barrière pour l'efficacité du piégeage.

Sur marnes, Rey [45] a observé que le piégeage de sédiments marneux peut avoir lieu grâce à l'action de barrières et de couvertures végétales constituées d'herbacées et de sous-arbustes, essentiellement dans le lit des ravines. L'auteur a également montré que, sur une zone marneuse en érosion de surface inférieure à $500 \mathrm{~m}^{2}$, un obstacle végétal recouvrant $20 \%$ de cette zone et situé entièrement à son aval peut permettre de maîtriser complètement la sortie de sédiments de celle-ci. Rovéra et al. [50] ont, quant à eux, pu observer un piégeage à l'amont de touffes herbacées, qui est fonction de la grosseur et de la densité des touffes. Le piégeage s'observe dès que la couverture végétale, composée d'herbacées et de ligneux bas, dépasse $50 \%$ de recouvrement au sol, jusqu'à des pentes de $30-35^{\circ}$. Avec une couverture encore plus dense, le piégeage est davantage pérenne (au-delà de trois ans).

Kikuchi et Miura [29] expliquent que les atterrissements créés par les obstacles végétaux sont favorables à l'installation d'une végétation colonisatrice, qui d'ailleurs ne parvient souvent à s'installer que sur ce type de dépôts relativement stables dans les bassins versants dégradés. Les végétaux constituant l'obstacle végétal peuvent également se développer au contact des sédiments atterris arrivant au niveau de l'obstacle après chaque phase de ruissellement, colonisant ainsi les sédiments piégés. La plus grande profondeur du sol et sa plus grande infiltrabilité sont généralement évoquées. Cette dynamique végétale naturelle peut permettre d'une part de retenir durablement les sédiments piégés grâce au développement des systèmes radiculaires, d'autre part d'augmenter la capacité de piégeage des obstacles végétaux [45].

En conséquence, à l'intérieur des bassins versants, de grandes quantités de sédiments érodés sont piégées et ne rejoignent pas l'exutoire des bassins $[8,19]$. 


\section{Efficacité des différentes formations végétales}

L'effet de la végétation peut être différent selon les formations végétales; il peut dépendre du type de végétation ou de l'utilisation du sol. Certains auteurs affirment que la forêt constitue la meilleure protection contre l'érosion hydrique de surface en montagne [30]. D'autres auteurs ont observé qu'une couverture arbustive ou une végétation basse peut offrir une protection similaire à celle jouée par des arbres [22]. Hill et Peart [26] ont également montré l'importance des strates végétales vis-à-vis de l'efficacité de la maîtrise de l'érosion. Ils ont ainsi observé une érosion croissante depuis une formation arborée et arbustive, une formation arborée, une formation uniquement au sol (herbacée et sous-arbustive), une formation au sol cultivée et enfin jusqu'aux sols nus. Sur marnes, Rovéra et al. [50] ont observé l'effet modérateur du couvert végétal en fonction de sa densité et de sa structure verticale. L'érosion diminue en effet lorsqu' on passe progressivement des marnes dénudées à un couvert d'arbres isolés, puis à un couvert arbustif et herbacé, et enfin à un couvert de pins groupés.

Le rôle de la couverture herbacée est souvent dénigré, alors que, bien souvent, elle peut être très efficace pour lutter contre l'érosion hydrique de surface. L'herbe - ou le gazon - présente notamment la particularité de bien recouvrir le sol et ainsi d'offrir de bonnes possibilités de stockage d'eau en profondeur [18]. Son action peut être optimale en mélange avec une formation arborée, les arbres permettant de dessécher les couches profondes, tandis que les herbes maintiennent la couche superficielle du sol. Dans les géofaciès où les ligneux n'ont pas lieu d'être (pistes de ski), ou ceux où les arbres sont à proscrire (grands mouvements de terrain ou certaines berges de cours d'eau), l'herbe peut représenter la solution adéquate. Certains auteurs [58] ont même observé une augmentation de l'érosion sur des terrains où une couverture herbacée avait été remplacée par une couverture arbustive. Ils expliquent cela par une moindre résistance des ligneux bas au ruissellement diffus. Toutefois, on doit constater qu'en contexte torrentiel, la couverture herbacée est bien souvent insuffisante pour lutter efficacement contre l'érosion concentrée. Il apparaît donc que, suivant le type de ruissellement, l'herbe ne constitue pas la meilleure solution pour lutter contre l'érosion linéaire.
Au sein d'une même strate végétale, l'efficacité de la protection contre l'érosion hydrique de surface peut donc dépendre des espèces végétales, notamment de leur morphologie et de leur dynamique [11]. En comparant l'effet de différents types de formations végétales, Sala et Calvo [51] concluent que la morphologie et la taille des végétaux sont importantes pour la mâ̂trise de l'érosion, car cela détermine la formation de litière, élément qui favorise l'infiltration de l'eau.

\section{Importance du taux de couverture végétale}

\subsection{Comparaison de terrains diversement végétalisés}

L'effet de la couverture végétale sur l'érosion hydrique de surface a pu être mis en évidence par comparaison entre des terrains diversement végétalisés. Il est souvent admis que, sur un versant partiellement végétalisé, les zones dénudées peuvent être soumises à l'érosion, alors que les zones végétalisées sont protégées [43]. Des études menées à l'échelle du bassin versant ont montré qu'en général l'érosion diminue quand la couverture végétale augmente [7]. Des résultats similaires ont été obtenus sur des surfaces plus réduites, celles du versant de quelques ares par exemple [12].

On a observé que l'érosion augmentait avec le pâturage [49], responsable d'une disparition partielle de la couverture végétale et d'un tassement des sols selon leur granulométrie. Heathwaite et al. [25] expliquent qu'un pâturage intensif peut mener à une réduction de l'infiltration de l'eau dans les sols de plus de $80 \%$, augmentant ainsi le ruissellement d'un rapport de 1 à 12 par rapport à des terrains similaires non pâturés.

La densité de la couverture végétale permet en général de déterminer s'il y a ruissellement ou pas : jusqu'à $70 \%$ de couverture végétale, l'eau ruisselle en grande partie; au-delà, l'eau percole et ne ruisselle plus, sauf s'il y a saturation des sols. En dessous de $70 \%$, la répartition de la végétation joue un rôle : pour une même densité, une répartition homogène de la végétation permet de maintenir un ruissellement diffus, alors qu'une répartition par plaques peut favoriser la concentration du ruissellement [27].

Plusieurs études ont montré que la production sédimentaire de bassins ou de versants diversement végétalisés ne variait pas de façon linéaire avec la couverture végétale. Ainsi, Rogers et Schumm [47] ont 
observé, sur des versants de pente $5^{\circ}$, que la production sédimentaire des versants augmentait rapidement lorsqu'on passait d'une couverture végétale de 43 à $15 \%$, puis que la production sédimentaire variait très peu pour des valeurs de couverture végétale inférieures à $15 \%$. Il n'y avait donc pas de relation linéaire ou exponentielle, et $15 \%$ apparaissait comme une valeur seuil minimale pour que la végétation joue un rôle significatif contre l'érosion. Les comparaisons des transports solides de deux bassins marneux diversement végétalisés ont également montré cette nonlinéarité de l'influence du couvert forestier sur la production de sédiments aux exutoires; on observe en effet une production spécifique annuelle de sédiments 220 fois moins importante dans un bassin versant végétalisé à $87 \%$ que dans un bassin versant végétalisé à $32 \%$ [46]. À l'échelle de ravines marneuses de surface inférieure à un hectare, Rey [44] a montré que la distribution spatiale de la végétation au sein de ces ravines est importante pour la réduction de la production sédimentaire à leur exutoire, en raison de l'existence de processus de piégeage des sédiments marneux par des barrières ou couvertures végétales. Des ravines de même couverture végétale totale peuvent avoir des productions sédimentaires très différentes et il est possible d'obtenir une production sédimentaire nulle à l'exutoire d'une ravine, avec seulement $33 \%$ de couverture végétale. Une valeur de $40 \%$ a été avancée par McIvor et al. [34] comme couverture végétale minimale à maintenir ou installer sur des terrains érodables pour une maîtrise significative de l'érosion. Enfin, Brochot [10] rapporte des faits montrant que l'enlèvement d'un quart de la forêt sur un bassin versant peut produire des effets très différents selon le degré de morcellement de la surface concernée, ainsi que de la position topographique des zones exploitées.

\subsection{Comparaisons après disparition de la couverture végétale}

Une augmentation de l'érosion hydrique de surface est généralement observée dans des bassins versants végétalisés qui ont été soumis à un incendie ayant provoqué la disparition de la couverture végétale [6]. Même sur des surfaces très réduites, le feu peut découvrir le sol et le rendre sensible aux reprises d'érosion. Ce processus érosif est surtout sensible pendant les premières années qui suivent l'incendie. À la suite d'un brûlage dirigé, une augmentation de l'érosion a également pu être observée par Soto et al. [54].

Les opérations de coupe forestière peuvent également être responsables d'une augmentation de l'érosion hydrique de surface, du fait de la suppression d'une partie de la biomasse protectrice et du fait de l'impact des travaux d'exploitation sur la structure des sols. Prosser et Soufi [42] soulignent que les potentialités de reprise d'érosion sont très fortes la première année après la coupe, pour des pluies journalières dépassant $80 \mathrm{~mm}$. Dans des bassins versants couverts par une forêt, Miller et al. [36] ont observé une forte recrudescence de l'érosion après une coupe rase. Ammer et al. [3] ont montré que des coupes partielles, sur 30 à $40 \%$ de la surface des bassins versants, entraînaient également une augmentation de la production sédimentaire. Des effets similaires ont été observés après l'enlèvement de formations végétales diverses dans des bassins versants, à la suite d'opérations de défrichement. Toutefois, Edeso et al. [21] expliquent que l'impact des coupes forestières sur l'érosion peut être minimisé avec des pratiques sylvicoles appropriées. Lacey [31] explique que des bandes forestières horizontales de $10 \mathrm{~m}$ de large laissées en l'état sur un versant peuvent permettre de réduire de $95 \%$ la production sédimentaire des terrains exploités. Keim et Schoenholtz [28] ont cependant observé que de telles bandes pouvaient parfois être inefficaces pour stopper l'érosion concentrée, initiée plus en amont. Certains auteurs expliquent d'ailleurs que, lors des exploitations forestières, ce sont surtout les ouvertures de pistes forestières qui sont responsables de l'activation de l'érosion sur les terrains érodables [16].

Les perturbations naturelles telles que les chablis peuvent également entraîner une apparition de processus d'érosion [5].

\subsection{Comparaisons après revégétalisation}

Les travaux de revégétalisation entraînent généralement une diminution de l'érosion hydrique de surface [46]. La plupart des auteurs attribuent cet effet de la végétation à l'augmentation de l'infiltration [59]. L'installation naturelle d'une couverture végétale sur des sols dégradés peut également mener à une diminution de l'érosion [38].

Toutefois, si les milieux revégétalisés permettent de réduire l'érosion par rapport à des milieux dégra- 
dés, ils ne restent cependant que des solutions intermédiaires, dont le degré de protection n'atteint pas celui offert par une végétation naturelle [4]. L'effet des plantations seules est parfois nettement limité, comme cela a pu être observé par Vacca et al. [57] dans une plantation d'eucalyptus.

\section{Conclusion}

Les nombreuses références bibliographiques présentées dans cet article plaident en faveur d'un rôle important joué par la végétation contre l'érosion hydrique de surface. Elles soulignent l'importance, audelà de la simple prise en compte du couvert végétal total, de la répartition du couvert végétal sur le terrain, du type de couvert et du type d'action de la végétation sur les différents processus érosifs.

Cette efficacité de la végétation peut être mise à profit dans la lutte contre les phénomènes érosifs. Cependant, l'installation d'une couverture végétale sur des terrains érodés doit faire appel à des techniques de génie biologique (par exemple, [53]). L'efficacité de ces techniques est elle-même abondamment étudiée par la communauté scientifique.

\section{Références}

[1] B. Abernethy, I.D. Rutherfurd, The effect of riparian tree roots on the mass-stability of riverbanks, Earth Surface Processes Landforms 25 (2000) 921-937.

[2] M. Abu-Zreig, R.P. Rudra, H.R. Whiteley, Validation of a vegetated filter strip model (VFSMOD), Hydrol. Processes 15 (2001) 729-742.

[3] U. Ammer, J. Breitsameter, J. Zander, Der Beitrag des Bergwaldes zum Schutz gegen Oberflächenabfluss und Bodenabtrag, Forstw. Cbl. 114 (1995) 232-249.

[4] V. Andreu, J.L. Rubio, E. Gimeno-Garcia, J.V. Llinares, Testing three Mediterranean shrub species in runoff reduction and sediment transport, Soil Tillage Res. 45 (1998) 441-454.

[5] O. Arnalds, The Icelandic 'rofabard' soil erosion features, Earth Surface Processes Landforms 25 (2000) 17-28.

[6] J.-L. Ballais, L'érosion consécutive à l'incendie d'août 1989 sur la montagne Sainte-Victoire : trois années d'observations (1989-1992), Bull. Association Géographie Fr. 70 (1993) 423437.

[7] M.C. Battany, M.E. Grismer, Rainfall runoff and erosion in Napa Valley vineyards: effects of slope, cover and surface roughness, Hydrol. Processes 14 (2000) 1289-1304.

[8] L. Beuselinck, A. Steegen, G. Govers, J. Nachtergaele, I. Takken, J. Poesen, Characteristics of sediment deposits formed by intense rainfall events in small catchments in the Belgian Loam Belt, Geomorphology 32 (2000) 69-82.
[9] E. Bochet, J. Poesen, J.L. Rubio, Mound development as an interaction of individual plants with soil, water erosion and sedimentation processes on slopes, Earth Surface Processes Landforms 25 (2000) 847-867.

[10] S. Brochot, Érosion de badlands dans le système DuranceÉtang de Berre, Cemagref-Agence de l'eau Rhône-Méditerranée-Corse, Grenoble, 1993, 7 fascicules.

[11] A.G. Brown, Soil erosion and fire in areas of Mediterraneantype vegetation: results from chaparral in southern California, USA and matorral in Andalucia, southern Spain, in: J.B. Thornes (Ed.), Vegetation and Erosion: Processes and Environments, Wiley, Chichester, UK, 1990, pp. 269-287.

[12] C. Carroll, L. Merton, P. Burger, Impact of vegetative cover and slope on runoff, erosion, and water quality for field plots on a range of soil and spoil materials on central Queensland coal mines, Aust. J. Soil Res. 38 (2000) 313-327.

[13] A. Cerdà, The influence of aspect and vegetation on seasonal changes in erosion under rainfall simulation on a clay soil in Spain, Can. J. Soil Sci. 78 (1998) 321-330.

[14] F. Combes, A. Hurand, M. Meunier, La forêt de montagne : un remède aux crues, in : Compte rendu de recherches $n^{\circ} 3, \mathrm{BVRE}$ de Draix, Cemagref Éditions, Grenoble, 1995, pp. 113-121.

[15] C. Cosandey, J.-F. Didon-Lescot, C. Martin, Forêt et écoulements : étude des processus responsables des modifications du bilan d'écoulement annuel à l'occasion d'une coupe forestière, Forêt méditerranéenne 21 (2000) 154-155.

[16] J. Croke, P. Hairsine, P. Fogarty, Sediment transport, redistribution and storage on logged forest hillslopes in south-eastern Australia, Hydrol. Processes 13 (1999) 2705-2720.

[17] P.A. Dalton, R.J. Smith, P.N.V. Truong, Vetiver grass hedges for erosion control on a cropped flood plain: hedge hydraulics, Agric. Water Manage. 31 (1996) 91-104.

[18] A. Derouiche, J. Bellot-Abad, D. Cartagena, Effet du couvert végétal sur le comportement hydrique et le transport solide dans une pinède à sous-bois arbustif et herbacé, Bull. Réseau Erosion 17 (1997) 24-36.

[19] D. Diallo, D. Orange, E. Roose, A. Morel, Potentiel de production de sédiments dans le bassin versant de Djitiko $\left(103 \mathrm{~km}^{2}\right)$, zone soudanienne du Mali Sud, Bull. Réseau Erosion 20 (2000) 54-66.

[20] T. Dunne, W. Zhang, B.F. Aubry, Effects of rainfall, vegetation, and microtopography on infiltration and runoff, Water Resour. Res. 27 (1991) 2271-2285.

[21] J.M. Edeso, A. Merino, M.J. Gonzalez, P. Marauri, Soil erosion under different harvesting management in steep forestlands from northern Spain, Land Degrad. Dev. 10 (1999) 79-88.

[22] C.F. Francis, J.B. Thornes, Runoff hydrographs from three Mediterranean vegetation cover types, in: J.B. Thornes (Ed.), Vegetation and Erosion: Processes and Environments, Wiley, Chichester, 1990, pp. 363-384.

[23] N. Geddes, D. Dunkerley, The influence of organic litter on the erosive effects of raindrops and of gravity drops released from desert shrubs, Catena 36 (1999) 303-313.

[24] A. Giordano, L'érosion et la lutte contre l'érosion en forêt méditerranéenne, Forêt méditerranéenne 15 (1994) 12-21.

[25] A.L. Heathwaite, T.P. Burt, S.T. Trudgill, The effect of land use on nitrogen, phosphorus and suspended sediment delivery 
to streams in a small catchment in southwest England, in : J.B. Thornes (Ed.), Vegetation and Erosion: Processes and Environments, Wiley, Chichester, 1990, pp. 161-177.

[26] R.D. Hill, M.R. Peart, Land use, runoff, erosion and their control: a review for southern China, Hydrol. Processes 12 (1998) 2029-2042.

[27] T. Ionesco, Considérations générales concernant les relations entre l'érosion et la végétation du Maroc, Rev. Géogr. Maroc 6 (1964) 17-28.

[28] R.F. Keim, S.H. Schoenholtz, Functions and effectiveness of silvicultural streamside management zones in loessial bluff forests, Forest Ecol. Manage. 118 (1999) 197-209.

[29] T. Kikuchi, O. Miura, Vegetation patterns in relation to microscale landforms in hilly land regions, Vegetatio 106 (1993) 147-154.

[30] K. Kim, Y. Jeong, Hydrological variations of discharge, soil loss and recession coefficient in three small forested catchments, Environ. Forest Sci. 54 (1998) 431-438

[31] S.T. Lacey, Runoff and sediment attenuation by undisturbed and lightly disturbed forest buffers, Water Air Soil Pollut. 122 (2000) 121-138.

[32] J. Lavabre, V. Andreassian, La forêt : un outil de gestion des eaux ?, Cemagref Editions, Paris, 2000.

[33] K.H. Lee, T.M. Isenhart, R.C. Schultz, S.K. Mickelson, Nutrient and sediment removal by switchgrass and cool-season grass filter strips in Central Iowa, USA, Agrofor. Syst. 44 (1999) 121-132

[34] J.G. McIvor, J. Williams, C.J. Gardener, Pasture management influences runoff and soil movement in the semi-arids tropics, Aust. J. Exp. Agric. 35 (1995) 55-65.

[35] L.D. Meyer, S.M. Dabney, W.C. Harmon, Sediment-trapping effectiveness of stiff-grass hedges, Trans. ASAE 38 (1995) 809-815.

[36] E.L. Miller, R.S. Beasley, E.R. Lawson, Forest harvest and site preparation effects on erosion and sedimentation in the Ouachita Mountains, J. Environ. Qual. 17 (1988) 219-225.

[37] C. O'Loughlin, Z. Xinbao, The influence of fast-growing conifer plantations on shallow landsliding and earthflow movement in New Zealand steeplands, in : Proc. 18th IUFRO World Congress, Ljubljana, Yugoslavia, IUFRO, 1986.

[38] G.A. Olyphant, D. Harper, Effects of direct revegetation on the hydrology, erosion and sediment yield of an abandoned deposit of coal-mine refuse, Geomorphology 11 (1995) 261-272.

[39] D. Pimentel, World Soil Erosion and Conservation, Cambridge University Press, Cambridge, 1993.

[40] J. Poesen, Gully erosion and environmental change: importance and research needs, Catena 50 (2003) 91-133.

[41] A. Poncet, Restauration et conservation des terrains en montagne, Office national des forêts, Paris, 1995.

[42] I.P. Prosser, M. Soufi, Controls on gully formation following forest clearing in a humid temperate environment, Water Resour. Res. 34 (1998) 3661-3671.

[43] K.D. Reid, B.P. Wilcox, D.D. Breshears, L. MacDonald, Runoff and erosion in a Pinon-Juniper woodland: influence of vegetation patches, Soil Sci. Soc. Am. J. 63 (1999) 1869-1879.

[44] F. Rey, Influence of vegetation distribution on sediment yield in forested marly gullies, Catena 50 (2003) 549-562.
[45] F. Rey, Effectiveness of vegetation barriers for marly sediment trapping, Earth Surf. Processes Landforms (2004).

[46] D. Richard, N. Mathys, Historique, contexte technique et scientifique des BVRE de Draix. Caractéristiques, données disponibles et principaux résultats acquis au cours de dix ans de suivi, in: Actes du colloque «Les bassins versants expérimentaux de Draix, laboratoire d'étude de l'érosion en montagne », Draix, Le Brusquet, Digne, France, 22-24 octobre 1997, Cemagref, Grenoble, France, 1999.

[47] R.D. Rogers, S.A. Schumm, The effect of sparse vegetative cover on erosion and sediment yield, J. Hydrol. 123 (1991) 1924.

[48] E. Roose, R. Bertrand, Contribution à l'étude de la méthode des bandes d'arrêt pour lutter contre l'érosion hydrique en Afrique de l'Ouest : résultats expérimentaux et observations sur le terrain, L'Agronomie tropicale 26 (1971) 12701283.

[49] E. Roose, M. Sabir, Stratégies traditionnelles de conservation de l'eau et des sols dans le bassin méditerranéen : classification en vue d'un usage renouvelé, Bull. Réseau Erosion 21 (2002) 33-44.

[50] G. Rovéra, Y. Robert, M. Coubat, R. Nedjaï, Érosion et stades biorhexistasiques dans les ravines du Saignon (Alpes de Provence) ; essai de modélisation statistique des vitesses d'érosion sur marnes, Étud. Géogr. Phys. 28 (1999) 109-115.

[51] M. Sala, A. Calvo, Response of four different Mediterranean vegetation types to runoff end erosion, in : J.B. Thornes (Ed.), Vegetation and Erosion: Processes and Environments, Wiley, Chichester, UK, 1990, pp. 347-362.

[52] K. Sassa, The mechanism of debris flows and the forest effect on their prevention, in: 18th IUFRO World Congress, Ljubljana, Yugoslavia, IUFRO, 1986.

[53] H.M. Schiechtl, R. Stern, Ground Bioengineering Techniques for Slope Protection and Erosion Control, Blackwell Science, Oxford, UK, 1996.

[54] B. Soto, R. Basanta, R. Perez, F. Diaz-Fierros, An experimental study of the influence of traditional slash-and-burn practices on soil erosion, Catena 24 (1995) 13-23.

[55] T. Stott, A comparison of stream bank erosion processes on forested and moorland streams in the Balquhidder Catchments, central Scotland, Earth Surface Processes Landforms 22 (1997) 383-399.

[56] C.R. Thorne, Effects of vegetation on riverbank erosion and stability, in: J.B. Thornes (Ed.), Vegetation and Erosion: Processes and Environments, Wiley, Chichester, 1990, pp. 125-143.

[57] A. Vacca, S. Loddo, G. Ollesch, R. Puddu, G. Serra, D. Tomasi, A. Aru, Measurement of runoff and soil erosion in three areas under different land use in Sardinia (Italy), Catena 40 (2000) 69-92.

[58] C.S. White, S.R. Loftin, Response of two semiarid grasslands to cool-season prescribed fire, J. Range Manage. 53 (2000) 52-61.

[59] A.D. Ziegler, T.W. Giambelluca, Influence of revegetation efforts on hydrologic response and erosion, Kaho'Olawe Island, Hawaii, Land Degrad. Dev. 9 (1998) 189-206.

[60] M. Zordia, Lutte contre les inondations par la reforestation, 1977. 